\section{JTI}

JOURNAL OF

TRAUMA AND INJURY

\title{
Successful Management of a Comatose Patient with Traumatic Brain Exposure with a Fronto-Parieto-Occipital Flap
}

\author{
Charles Chidiebele Maduba, F.W.A.C.S., \\ Ugochukwu Uzodimma Nnadozie, F.W.A.C.S \\ Division of Plastic Surgery, Department of Surgery, Alex Ekwueme Federal University \\ Teaching Hospital Abakaliki, Ebonyi State, Nigeria
}

Received: November 1, 2019

Revised: January 15, 2020

Accepted: January 29, 2020

\section{Correspondence to}

\section{Charles Chidiebele Maduba,}

\section{F.W.A.C.S}

Division of Plastic Surgery, Department of Surgery, Alex Ekwueme Federal University Teaching Hospital Abakaliki, P.M.B 102,

Ebonyi State, Nigeria

Tel: +234-80-3601-7909

E-mail:charlesmaduba@gmail.com
Composite skull defects in patients with severe head injuries are very challenging to manage. The dilemma when deciding whether to perform a definitive reconstruction is how long to wait for physiological recovery before an intervention complicates the situation. The inability of such patients to tolerate prolonged anesthetic exposure is a driving factor for performing the minimal intervention necessary to facilitate recovery. Herein, we present a case involving the successful immediate reconstructive treatment of a severely head-injured adolescent with a composite scalp defect secondary to trauma. A 14-year-old boy sustained a severe head injury from a motor vehicle accident with a composite scalp defect in the right fronto-parietal region. The frontal lobe was exposed, and the right eye was crushed and devitalized. The patient was deeply unconscious for 3 days, without any significant improvements before reconstructive surgery was proposed due to fear of possible meningitis resulting from the exposure of brain structures. We successfully managed the patient with a fronto-parieto-occipital flap, after which the patient promptly recovered consciousness.

Keywords: Composite scalp defect; Head injury; Flap; Unconsciousness

\section{INTRODUCTION}

Composite scalp defects are very challenging for both plastic surgeons and neurosurgeons. There is an urgent need to address such defects to protect the exposed brain and to prevent infection and other metabolic complications. However, the patient's general health status must be considered when proposing reconstructive options [1]. In the setting of trauma, the force that produced the defect is generally of sufficient (http://creativecommons.org/licenses/by-nc/4.0/) which permits unrestricted noncommercial use, distribution, and reproduction in any medium, provided the original work is properly cited. 
magnitude to cause significant injury to the brain parenchyma. Furthermore, traumatic mechanisms of injury cause contamination, which may result in brain infection. Given these issues, the principle of reconstruction in such cases is traditionally to employ the simplest and most reliable option [2].

Composite scalp defects expose the brain to both primary and secondary injuries, and meningitis is a feared complication. There is an urgent need to handle defects involving brain exposure to prevent infectious and metabolic challenges in the acute phase of the injury. However, whether the patient is sufficiently stable to undergo such an extensive procedure is often a major limiting factor. Therefore, the usual practice is to do as little as possible to minimize the duration of anesthesia.

There are many options to cover composite defects, including autogenous and artificial materials, both of which have specific advantages and disadvantages $[1,3]$. In the acute traumatic period, there is extensive wound con-

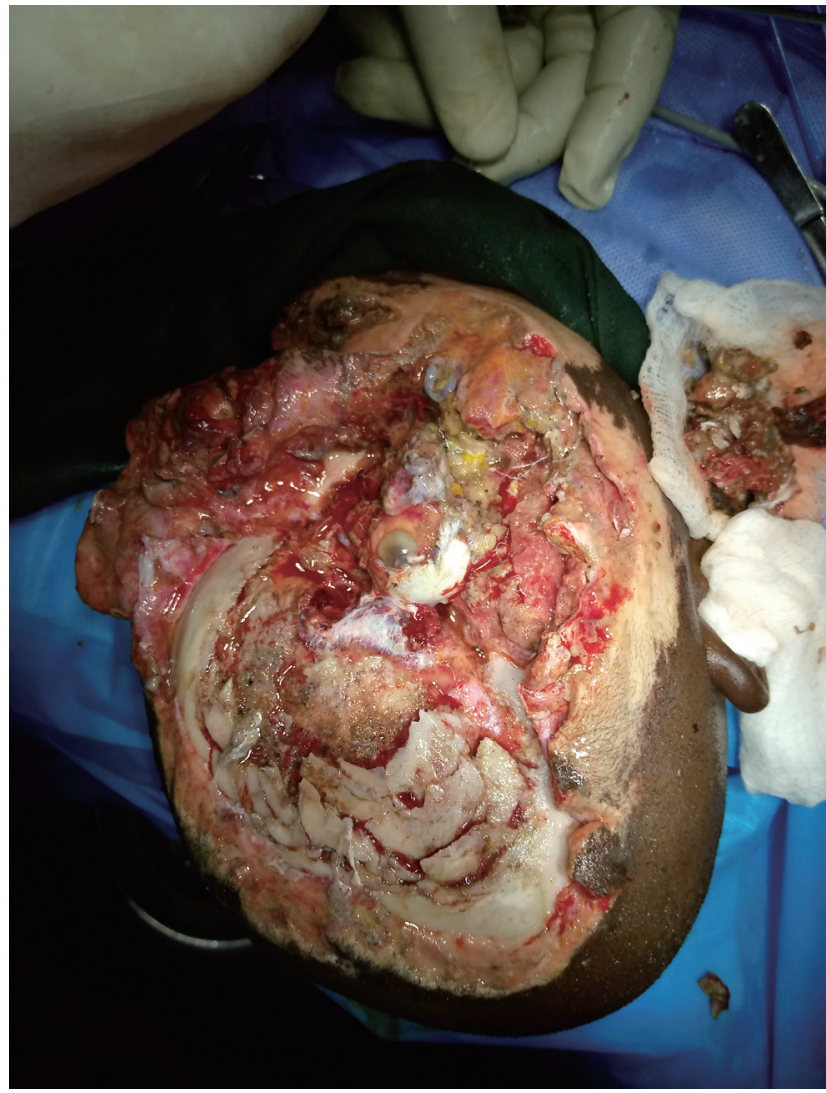

Fig. 1. The wound after debridement. tamination, which makes the use of artificial materials an unlikely option for coverage. Thus, it is often unavoidably necessary to use autogenous tissue for coverage.

The need to limit surgical time in deeply unconscious patients also restricts the feasibility of performing aggressive interventions, such as providing a bony cover as a skull equivalent. Therefore, in this case, we restricted ourselves to providing scalp flap coverage that was adequate for the exposed brain.

\section{CASE REPORT}

A 14-year-old boy presented with fronto-parietal composite scalp loss after being ejected from a motorized tricycle upon losing control at high speed and somersaulting a number of times. He lost consciousness and remained in a coma for 3 days before the surgical intervention. He had significant blood loss.

The examination showed an adolescent boy who was deeply unconscious, with the highest Glasgow Coma Scale score of 7. He was pale, afebrile, and had an oxygen saturation of $85-90 \%$ on room air and $100 \%$ on oxygen. He had no respiratory distress. His major tissue injuries included a right fronto-parietal osseocutaneous avulsion measuring approximately $12 \times 10 \mathrm{~cm}$ superiorly and $9 \times 7 \mathrm{~cm}$ inferiorly. The full-thickness cranial defect measured roughly $8 \times 5 \mathrm{~cm}$, with exposure of the contused

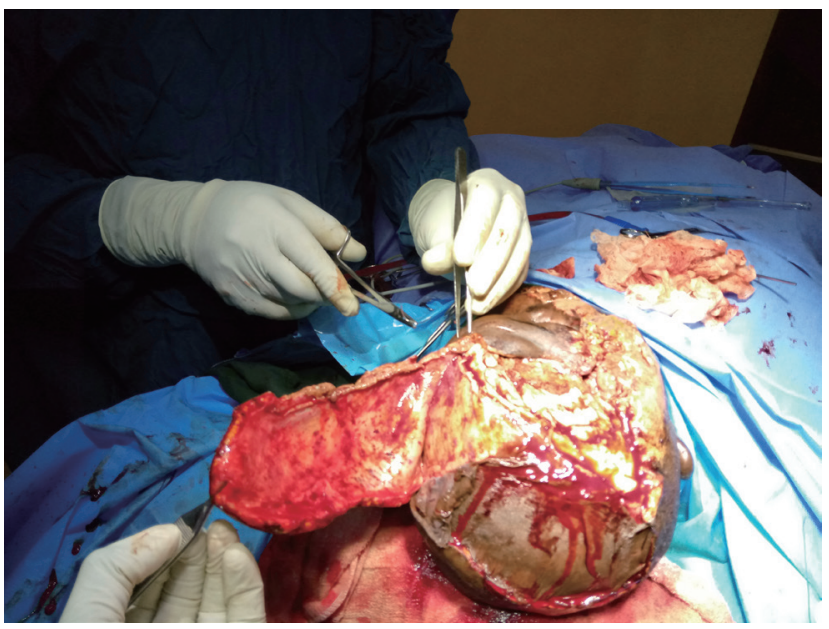

Fig. 2. A fronto-parieto-occipital flap was raised. 


\section{JTI}

right frontal lobe. An avulsion wound was also present on the right cheek and the patient's right eye was devascularized (Fig. 1). The patient had also sustained an avulsion wound on the right leg that was superficial to the deep fascia and measured approximately $8 \times 6 \mathrm{~cm}$.

The patient was assessed by the neurosurgical team upon presentation, after which the plastic surgery and ophthalmology teams were also consulted. The patient was diagnosed with multiple injuries, including a severe head injury, right eye loss, osseocutaneous fronto-parietal scalp loss, and soft tissue injuries to the cheek and extremity. A joint operative session was planned by the three surgical units. The need to protect the exposed brain with a short duration of anesthesia was discussed by the managing surgical teams with the anesthetists. The uncertainty of the outcome was also discussed with the patient's parents. The major challenge was financial, as the caregivers could not afford a computed tomography scan. The laboratory investigations done included serum electro-

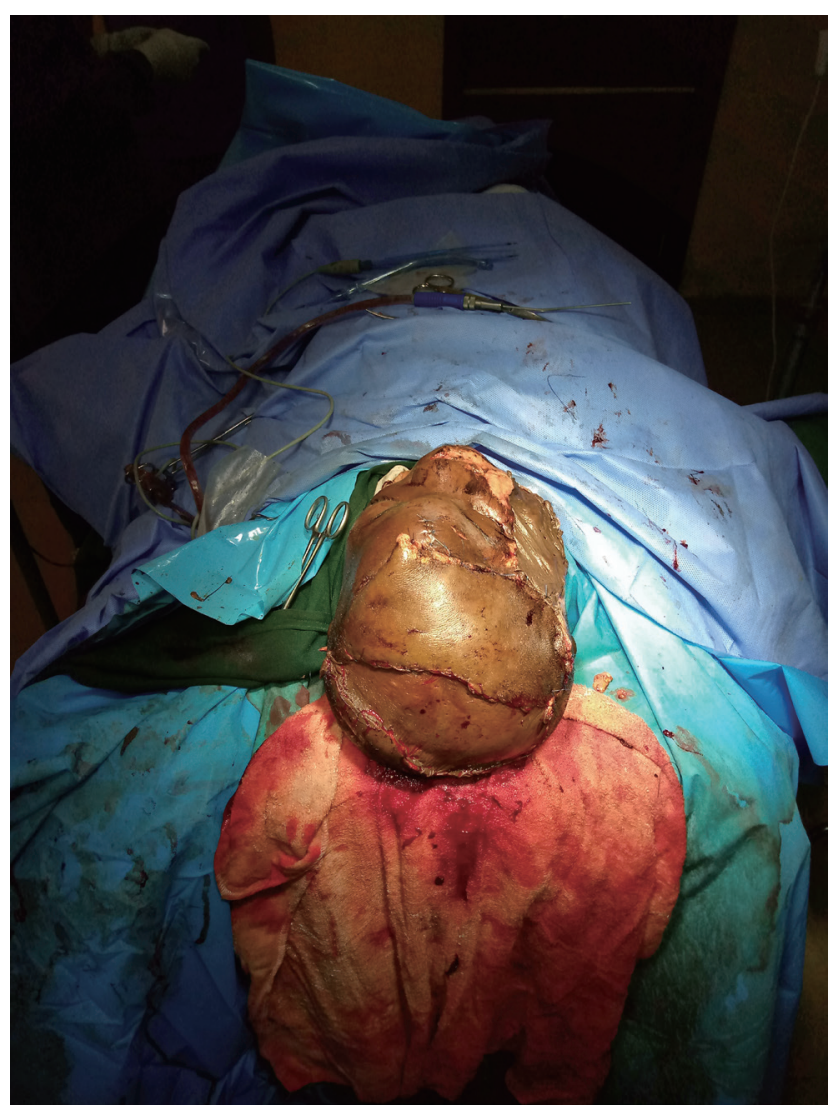

Fig. 3. Immediate post-reconstruction photograph. lytes, blood urea and creatinine, blood glucose estimation, packed cell volume, blood grouping, and cross-matching. The patient's hematocrit was $23 \%$, and three units of sedimented cells were transfused before and during surgery. The other laboratory parameters were within the normal range. Financial constraints also delayed the surgical intervention, as the patient had no health insurance coverage and the parents had to pay for the procedure out of pocket. In the ensuing 3 days, the patient had alternating wound dressings with $5 \%$ povidone-iodine-soaked gauze and moderately firm bandage application.

The patient was taken to the operating theater on day 3 post-injury. Under general anesthesia with endotracheal intubation, in the supine position, the surgical site was prepared aseptically and the patient was covered with sterile drapes. Wound debridement was done and the devascularized dead right eye was enucleated by the

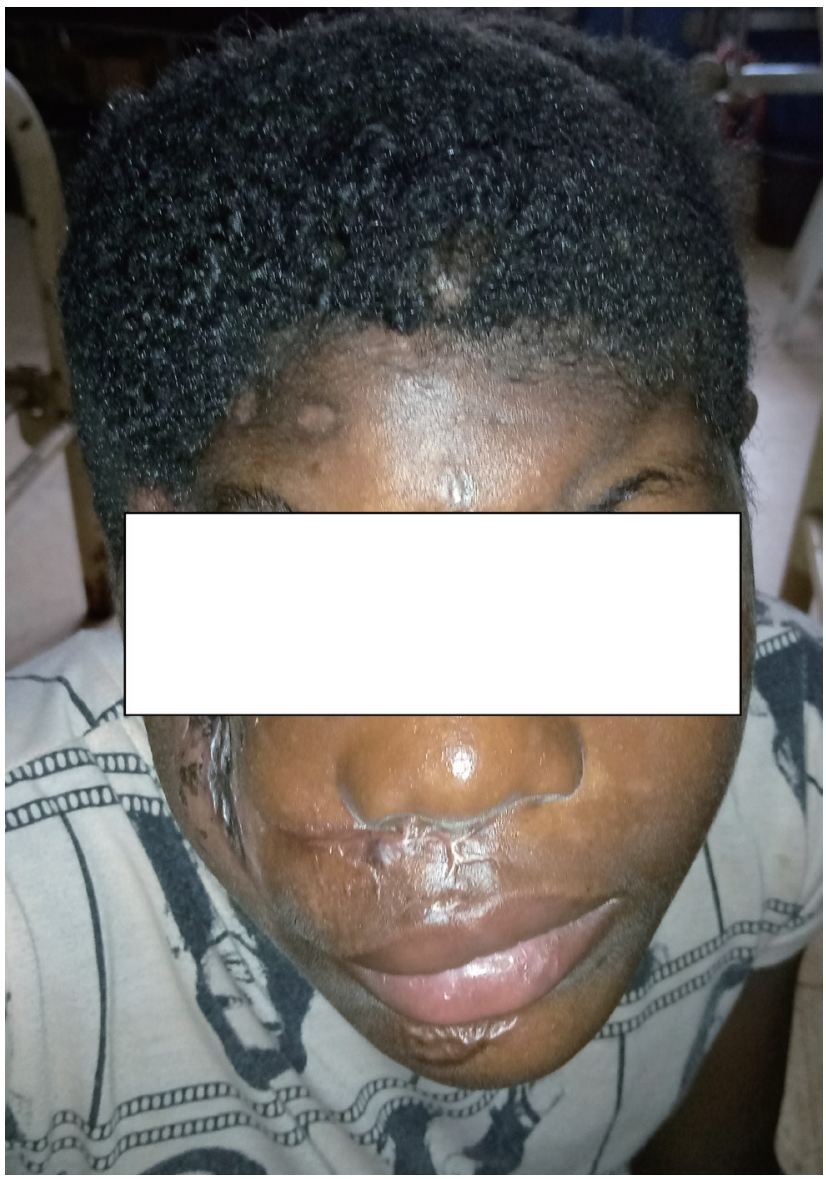

Fig. 4. Photograph taken 3 months after reconstruction. 
ophthalmologist. A left fronto-parieto-temporal scalp flap was planned in reverse, raised, and transferred to the right fronto-parietal wound, completely covering the exposed contused brain. The secondary scalp defect was covered with a split-thickness skin graft. The cheek wound was also covered with a split-thickness skin graft. Conventional gauze dressing was used for both the flap and split-thickness skin graft recipient wound sites and for the split-thickness skin graft donor site.

The patient's postoperative management included parenteral presumptive antibiotics since the wound was grossly contaminated, analgesics, intravenous fluid administration, nasogastric tube feeding, and wound dressing. Broad-spectrum antibiotics were chosen; anaerobes were covered with parenteral metronidazole and aerobes (especially Gram-negative bacteria) were covered with parenteral ceftriaxone. Intravenous metronidazole was given at a dose of $500 \mathrm{mg}$ every 8 hours, while intravenous ceftriaxone was given at a dose of $1 \mathrm{~g}$ at 12-hour intervals; both antibiotics were continued for 1 week. There was $100 \%$ flap survival and $100 \%$ graft take at the secondary defect on the scalp. Graft take on the right cheek wound was about $65 \%$.

Recovery of consciousness progressed gradually. On postoperative day 2, the patient's Glasgow Coma Score had improved to 10 , and it further increased to 13 on day 3. He regained full consciousness on postoperative day 4. Flap and graft healing and consolidation continued until roughly 5 weeks after surgery, when the patient was discharged home to continue follow-up on an outpatient basis.

\section{DISCUSSION}

High-impact traumatic brain injuries, when associated with composite scalp loss, cause significant primary and potentially fatal secondary brain insults. Infection is a major secondary event after traumatic brain injuries [4]. In this case, surgery for early wound coverage aimed to prevent infection and to facilitate metabolic recovery, while providing a protective covering for the brain. The dilemma either to operate or to wait to observe outcomes in the surgical theater is heightened by the cardiopulmonary and metabolic instability of such patients resulting from their response to trauma.

Our patient was deeply unconscious and had initial cardiopulmonary instability, which stabilized following resuscitation. The need to prevent secondary bacterial infection of the brain informed the choice to perform early flap coverage despite the severe brain injury. Simpler techniques of reconstruction, such as split-thickness skin grafting, were not used because the bed was inappropriate (i.e., it was necessary to place the graft on bare bone in one part and on exposed brain parenchyma in the other part).
A

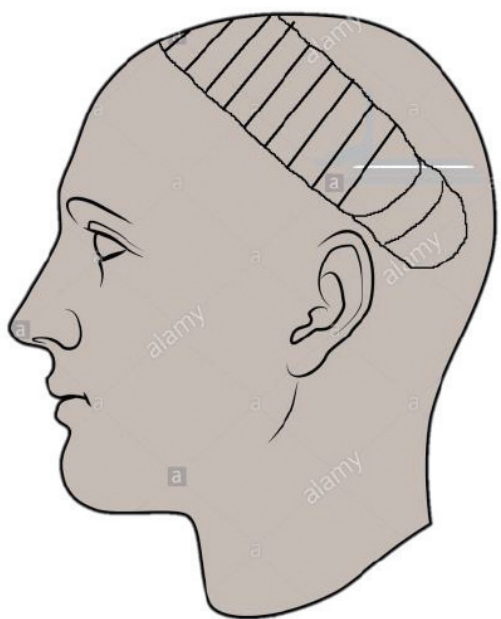

B

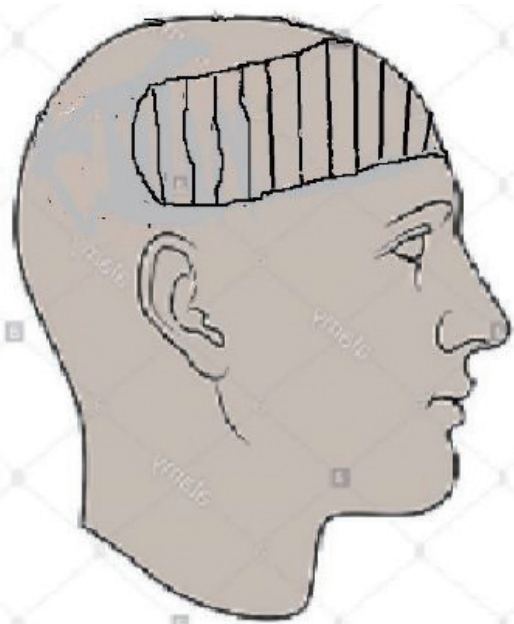

Fig. 5. Schematic diagram of the right fronto-parieto-occipital flap. (A) Left fronto-parieto-occipital flap pre-elevation. (B) Right fronto-parietal flap inset. 
A contralateral fronto-parieto-occipital flap based on the frontal artery was chosen (Fig. 2) [5]. This is a versatile flap that is capable of achieving relatively wide coverage and has excellent survival outcomes. It is raised on the sub-galeal plane. The secondary defect is usually covered with a split-thickness skin graft, as was done in our patient (Fig. 3).

The flap, with its blood supply, may contribute to the control of infection from the wound bed. Meningitis, mainly induced by pneumococci, is a potentially fatal complication of traumatic brain injuries $[5,6]$. It may be more likely in patients with traumatic composite scalp defects due to gross contamination and consequent sepsis [7].

The flap also protected the brain parenchyma from secondary trauma caused by the wound dressing and other secondary brain injuries. This may have contributed to the patient's rapid recovery of consciousness, with full consciousness restored by postoperative day 4 .

The quality of flap coverage was clinically satisfactory, progressively consolidating from a soft consistency after surgery to a firm consistency. There was a suspicion of possible neo-osteosynthesis under the flap, which provided a roof for the brain parenchyma (Fig. 4). This necessitated a late postoperative X-ray that was requested 12 weeks post-reconstruction. However, the patient was lost to follow-up and the requested imaging study could not be performed.

To the best of our knowledge, scalp reconstruction using this flap has not been previously reported in a severely head-injured patient in Sub-Saharan Africa. The most similar previous report dealt with a child with a moderate head injury who had a much smaller defect and was treated using a random scalp flap [8]. Therefore, we recommend the prompt use of this flap, even in patients in a coma, to reconstruct composite frontoparietal scalp defects (Fig. 5). Additional case reports or series would help to ascertain whether early coverage using this flap contributes to recovery from unconsciousness and brain function.

A composite scalp defect in a severely head-injured patient managed with a fronto-parieto-occipital flap showed early rapid recovery after flap coverage of exposed brain parenchyma. The flap protected the patient from secondary brain insults and may have contributed to an early recovery.

\section{REFERENCES}

1. Steiner D, Horch RE, Eyüpoglu I, Buchfelder M, Arkudas A, Schmitz M, et al. Reconstruction of composite defects of the scalp and neurocranium-a treatment algorithm from local flaps to combined AV loop free flap reconstruction. World J Surg Oncol 2018;16:217.

2. Lee B, Bickel K, Levin S. Microsurgical reconstruction of extensive scalp defects. J Reconstr Microsurg 1999;15:255-62.

3. Leedy JE, Janis JE, Rohrich RJ. Reconstruction of acquired scalp defects: an algorithmic approach. Plast Reconstr Surg 2005;116:54e-72.

4. Appelbaum E. Meningitis following trauma to the head and face. JAMA1960;173:1818-22.

5. Juri J. Diagonal fronto-parieto-occipital scalp flaps. In: Strauch B, et al. (eds). Grabb's encyclopedia of flaps. 3rd ed. Vol. 1; Philadelphia:Lippincott Williams \& Wilkins;2009:17-19.

6. Gallivan M, Murray E, Harriman K. Association of streptococcus pneumoniae meningitis after head injury or brain surgery-California, 2013-2014. Open Forum Infectious Diseases 2015;2(Supp 1):1896.

7. Kaptigau WM, Ke L, Rosenfeld JV. Open depressed and penetrating skull fractures in Port Moresby General Hospital from 2003 to 2005. P N G Med J 2007;50:58-63.

8. Kim Y, Morris MC, Lee TC, Earnest RE. Surgical management of compound skull fracture with exposed brain matter in a third-world country. J Surg Case Rep 2019;2019:rjz147. 